\title{
Correction to: Kurdyka-Łojasiewicz Property of Zero-Norm Composite Functions
}

\author{
Yuqia $\mathrm{Wu}^{1} \cdot$ Shaohua $\operatorname{Pan}^{1} \cdot$ Shujun $\mathrm{Bi}^{1}$
}

Received: 27 January 2021 / Accepted: 2 April 2021 / Published online: 6 May 2021

c Springer Science+Business Media, LLC, part of Springer Nature 2021

\section{Correction to: Journal of Optimization Theory and Applications (2021) 188:94-112 https://doi.org/10.1007/s10957-020-01779-7}

\section{Introduction}

In our paper [6], there is a gap for the statement of Proposition 3.2 and Remark 3.2. In addition, on line 14 of page 110 , the set $\left[x^{\prime} \in C, \operatorname{supp}\left(x^{\prime}\right)=J, x^{\prime} \rightarrow \bar{x}, x^{\prime} \neq \bar{x}\right]$ may be empty. In this erratum, we provide the correct statements for Proposition 3.2 and Remark 3.2 and update the proof of Proposition 3.2.

\section{Corrected Result}

First, we give the correct statement of [6, Proposition $3.2 \&$ Remark 3.2].

Proposition 2.1 ([6, Proposition 3.2] corrected) (i) When $h(\cdot)=v\|\cdot\|_{0}$ for a constant $v>0$, if $\left.\left.\psi: \mathbb{R}^{p} \rightarrow\right]-\infty,+\infty\right]$ is a proper closed piecewise linear regular function,

\section{Communicated by Boris S. Mordukhovich.}

The original article can be found online at https://doi.org/10.1007/s10957-020-01779-7.

Shujun Bi

bishj@scut.edu.cn

Yuqia Wu

math_wuyuqia@mail.scut.edu.cn

Shaohua Pan

shhpan@scut.edu.cn

1 School of Mathematics, South China University of Technology, Guangzhou, China 
then for any $\bar{x} \in \operatorname{dom} \psi$,

$$
\begin{aligned}
\widehat{\partial}(\psi+h)(\bar{x}) & =\partial \psi(\bar{x})+\partial h(\bar{x})=\partial(\psi+h)(\bar{x}) \\
\partial^{\infty}(\psi+h)(\bar{x}) & =[\partial \psi(\bar{x})+\partial h(\bar{x})]^{\infty}
\end{aligned}
$$

if $\psi$ is an indicator function of some closed convex set $C \subseteq \mathbb{R}^{p}$, then for any $\bar{x} \in C$ such that $\left\{x \in \mathbb{R}^{p} \mid x_{i}=0\right.$ for $\left.i \notin \operatorname{supp}(\bar{x})\right\} \cap \operatorname{ri}(C) \neq \emptyset$, it holds that

$$
\widehat{\partial}(\psi+h)(\bar{x})=\partial \psi(\bar{x})+\partial h(\bar{x})=\partial(\psi+h)(\bar{x})=\partial^{\infty}(\psi+h)(\bar{x})=[\widehat{\partial}(\psi+h)(\bar{x})]^{\infty} .
$$

(ii) When $h=\delta_{\Omega}$, the indicator function of $\Omega:=\left\{x \in \mathbb{R}^{p}:\|x\|_{0} \leq \kappa\right\}$ for an integer $\kappa>0$, the results of part (i) hold at any $\bar{x} \in \operatorname{dom} \psi$ with $\|\bar{x}\|_{0}=\kappa$, and at any $\bar{x} \in \operatorname{dom} \psi$ with $\|\bar{x}\|_{0}<\kappa$ it holds that $\partial(\psi+h)(\bar{x}) \subseteq \partial \psi(x)+\partial h(\bar{x})$.

Remark 2.1 ([6, Remark 3.2] corrected) When $\psi$ is a locally Lipschitz regular function, the first part of Proposition 2.1 still holds by [5, Theorem 9.13(b) \& Corollary 10.9], and now equality (2) is also given in [2, Proposition 1.107(iii)] and [3, Prop. 1.29].

In what follows, we provide the proof of Proposition 2.1.

The proof of Proposition 2.1: First, we consider that $\psi$ is a proper closed piecewise linear regular function. Fix any $\bar{x} \in \operatorname{dom} \psi$. Notice that epi $\psi$ and epi $h$ are the union of finitely many polyhedral sets. By combining [4, Proposition 1] and [1, Section 3.2], it then follows that

$$
\partial(\psi+h)(\bar{x}) \subseteq \partial \psi(\bar{x})+\partial h(\bar{x}) \text { and } \partial^{\infty}(\psi+h)(\bar{x}) \subseteq \partial^{\infty} \psi(\bar{x})+\partial^{\infty} h(\bar{x})
$$

From the first inclusion, $\partial(\psi+h)(\bar{x}) \supseteq \widehat{\partial}(\psi+h)(\bar{x}) \supseteq \widehat{\partial} \psi(\bar{x})+\widehat{\partial} h(\bar{x})$, and the regularity of $\psi$ and $h$, we obtain the equalities in (1). When $\partial \psi(\bar{x})=\emptyset$, obviously, the equalities in (2) hold. So, it suffices to consider the case where $\partial \psi(\bar{x}) \neq \varnothing$. From the second inclusion in (3), it follows that

$$
\left[\partial^{\infty}(\psi+h)(\bar{x})\right]^{\circ} \supseteq\left[\partial^{\infty} \psi(\bar{x})+\partial^{\infty} h(\bar{x})\right]^{\circ}=\left[\partial^{\infty} \psi(\bar{x})\right]^{\circ} \cap\left[\partial^{\infty} h(\bar{x})\right]^{\circ}
$$

where $K^{\circ}$ denotes the negative polar of a cone $K$. By combining this inclusion with [5, Exercise 8.23] and the second equality of (1), for any $w \in \mathbb{R}^{p}$ we have

$$
\begin{aligned}
\widehat{d}(\psi+h)(\bar{x})(w) & \leq \widehat{d} \psi(\bar{x})(w)+\widehat{d h}(\bar{x})(w)=d \psi(\bar{x})(w)+d h(\bar{x})(w) \\
& \leq d(\psi+h)(\bar{x})(w) \leq \widehat{d}(\psi+h)(\bar{x})(w)
\end{aligned}
$$

where $\widehat{d} h(\bar{x})$ and $d h(\bar{x})$, respectively, denote the regular subderivative and the subderivative of $\psi+h$ at $\bar{x}$, the equality is due to the regularity of $\psi$ and $h$, and the second inequality is using [5, Corollary 10.9]. By [5, Corollary 8.19], this shows that $\psi+h$ is regular, and hence $\partial^{\infty}(\psi+h)(\bar{x})=[\widehat{\partial}(\psi+h)(\bar{x})]^{\infty}=[\partial \psi(\bar{x})+\partial h(\bar{x})]^{\infty}$. Thus, we obtain the first part. 
Next we consider the case $\psi=\delta_{C}$. Fix any $\bar{x} \in C$ with $\operatorname{ri}(C) \cap L_{\bar{x}} \neq \emptyset$, where $L_{\bar{x}}:=\left\{x \in \mathbb{R}^{p} \mid x_{i}=0\right.$ for $\left.i \notin \operatorname{supp}(\bar{x})\right\}$. Let $J=\operatorname{supp}(\bar{x})$. We first argue

$$
\widehat{\partial}\left(\delta_{C}+h\right)(\bar{x}) \subseteq \partial \delta_{C \cap L_{\bar{x}}}(\bar{x}) .
$$

If there exists $\varepsilon>0$ such that $[\mathbb{B}(\bar{x}, \varepsilon) \backslash\{\bar{x}\}] \cap\left[C \cap L_{\bar{x}}\right]=\emptyset$, then $\partial \delta_{C \cap L_{\bar{x}}}(\bar{x})=$ $\mathcal{N}_{C \cap L_{\bar{x}}}(\bar{x})=\mathbb{R}^{p}$, and the inclusion in (4) clearly holds. So, it suffices to consider that for any $\varepsilon>0,[\mathbb{B}(\bar{x}, \varepsilon) \backslash\{\bar{x}\}] \cap\left[C \cap L_{\bar{x}}\right] \neq \emptyset$. Pick any $v \in \widehat{\partial}\left(\delta_{C}+h\right)(\bar{x})$. By the definition of regular subgradient, it follows that

$$
\begin{aligned}
& 0 \leq \liminf _{\bar{x} \neq x^{\prime} \rightarrow \bar{x}} \frac{h\left(x^{\prime}\right)+\delta_{C}\left(x^{\prime}\right)-h(\bar{x})-\delta_{C}(\bar{x})-\left\langle v, x^{\prime}-\bar{x}\right\rangle}{\left\|x^{\prime}-\bar{x}\right\|}
\end{aligned}
$$

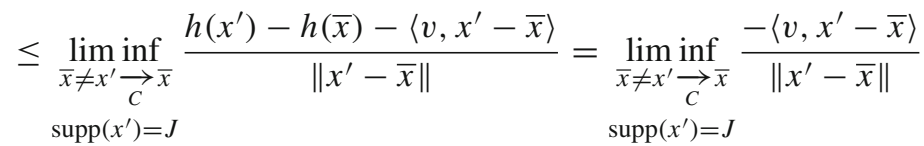

$$
\begin{aligned}
& \begin{aligned}
= & \liminf _{\bar{x} \neq x^{\prime} \underset{C}{\rightarrow} \bar{x}} \frac{\delta_{C \cap L_{\bar{x}}}\left(x^{\prime}\right)-\delta_{C \cap L_{\bar{x}}}(\bar{x})-\left\langle v, x^{\prime}-\bar{x}\right\rangle}{\left\|x^{\prime}-\bar{x}\right\|}, \\
& \operatorname{supp}\left(x^{\prime}\right)=J
\end{aligned}
\end{aligned}
$$

which implies that $v \in \widehat{\partial} \delta_{C \cap L_{\bar{x}}}(\bar{x})=\partial \delta_{C \cap L_{\bar{x}}}(\bar{x})$. The inclusion in (4) holds. By combining (4) with [5, Corollary 10.9] and $\partial h(\bar{x})=\mathcal{N}_{L_{\bar{x}}}(\bar{x})$, we have

$$
\begin{gathered}
\partial \delta_{C}(\bar{x})+\partial h(\bar{x})=\widehat{\partial} \delta_{C}(\bar{x})+\widehat{\partial} h(\bar{x}) \subseteq \widehat{\partial}\left(\delta_{C}+h\right)(\bar{x}) \subseteq \partial\left(\delta_{C}+\delta_{L_{\bar{x}}}\right)(\bar{x}) \\
=\partial \delta_{C}(\bar{x})+\partial \delta_{L_{\bar{x}}}(\bar{x})=\partial \delta_{C}(\bar{x})+\partial h(\bar{x}) .
\end{gathered}
$$

where the second equality is due to $\operatorname{ri} C \cap L_{\bar{x}} \neq \emptyset$. In fact, from the above arguments, we conclude that for all $x \in C$ with $\operatorname{ri}(C) \cap L_{x} \neq \emptyset$,

$$
\widehat{\partial}\left(\delta_{C}+h\right)(x)=\partial \delta_{C}(x)+\partial h(x)=\partial \delta_{C \cap L_{x}}(x) .
$$

Now we argue that $\partial\left(\delta_{C}+h\right)(\bar{x}) \subseteq \partial \delta_{C}(\bar{x})+\partial h(\bar{x})$. To this end, pick any $v \in$ $\partial\left(\delta_{C}+h\right)(\bar{x})$. Then, there exist sequences $x^{k} \underset{\delta_{C}+h}{\longrightarrow} \bar{x}$ and $v^{k} \in \widehat{\partial}\left(\delta_{C}+h\right)\left(x^{k}\right)$ with $v^{k} \rightarrow v$ as $k \rightarrow \infty$. Since $\delta_{C}\left(x^{k}\right)+h\left(x^{k}\right) \rightarrow \delta_{C}(\bar{x})+h(\bar{x})$, we must have $x^{k} \in C$ and $h\left(x^{k}\right) \rightarrow h(\bar{x})$ for all $k$ large enough. The latter, along with $\operatorname{supp}\left(x^{k}\right) \supseteq J$, implies that $\operatorname{supp}\left(x^{k}\right)=J$ for all sufficiently large $k$. By invoking (6), for all sufficiently large $k, v^{k} \in \partial \delta_{C}\left(x^{k}\right)+\partial h\left(x^{k}\right)$. By passing to the limit $k \rightarrow \infty$ and using $h\left(x^{k}\right) \rightarrow h(\bar{x})$, we obtain $v \in \partial \delta_{C}(\bar{x})+\partial h(\bar{x})$. By the arbitrariness of $v$ in $\partial\left(\delta_{C}+h\right)(\bar{x})$, the stated inclusion follows. In particular, together with $\partial\left(\delta_{C}+h\right)(\bar{x}) \supseteq \widehat{\partial}\left(\delta_{C}+h\right)(\bar{x})=\partial \delta_{C}(\bar{x})+$ $\partial h(\bar{x})$ and $(5)$,

$$
\widehat{\partial}\left(\delta_{C}+h\right)(\bar{x})=\partial\left(\delta_{C}+h\right)(\bar{x})=\mathcal{N}_{C}(\bar{x})+\partial h(\bar{x})=\partial \delta_{C \cap L_{\bar{x}}}(\bar{x}) .
$$

Next we argue $\partial^{\infty}\left(\delta_{C}+h\right)(\bar{x})=\partial^{\infty} \delta_{C \cap L_{\bar{x}}}(\bar{x})$. Pick any $u \in \partial^{\infty}\left(\delta_{C}+h\right)(\bar{x})$. Then, there exist sequences $x^{k} \underset{\delta_{C}+h}{\longrightarrow} \bar{x}$ and $u^{k} \in \widehat{\partial}\left(\delta_{C}+h\right)\left(x^{k}\right)$ with $\lambda_{k} u^{k} \rightarrow u$ for some 
$\lambda_{k} \downarrow 0$ as $k \rightarrow \infty$. By following the same arguments as above, $\operatorname{supp}\left(x^{k}\right)=J$ for all $k$ large enough. Together with (6) and $u^{k} \in \widehat{\partial}\left(\delta_{C}+h\right)\left(x^{k}\right)$, we have $u^{k} \in$ $\widehat{\partial} \delta_{C \cap L_{\bar{x}}}\left(x^{k}\right)$ for all $k$ large enough. Notice that $x^{k} \underset{C \cap L_{\bar{x}}}{\longrightarrow} \bar{x}$. So, $u \in \partial^{\infty}\left(\delta_{C}+\delta_{L_{\bar{x}}}\right)(\bar{x})$ and $\partial^{\infty}\left(\delta_{C}+h\right)(\bar{x}) \subseteq \partial^{\infty} \delta_{C \cap L_{\bar{x}}}(\bar{x})$. Conversely, pick any $u \in \partial^{\infty} \delta_{C \cap L_{\bar{x}}}(\bar{x})$. There exist $x^{k} \underset{C \cap L_{\bar{x}}}{\longrightarrow} \bar{x}$ and $u^{k} \in \widehat{\partial} \delta_{C \cap L_{\bar{x}}}\left(x^{k}\right)$ with $\lambda_{k} u^{k} \rightarrow u$ for some $\lambda_{k} \downarrow 0$ as $k \rightarrow \infty$. Clearly, $\left(\delta_{C}+h\right)\left(x^{k}\right) \rightarrow\left(\delta_{C}+h\right)(\bar{x})$. Also, from (6) and $u^{k} \in \widehat{\partial} \delta_{C \cap L_{\bar{x}}}\left(x^{k}\right)$, we have $u^{k} \in \widehat{\partial}\left(\delta_{C}+h\right)\left(x^{k}\right)$. So, $u \in \partial^{\infty}\left(\delta_{C}+h\right)(\bar{x})$, and $\partial^{\infty}\left(\delta_{C}+h\right)(\bar{x}) \supseteq \partial^{\infty} \delta_{C \cap L_{\bar{x}}}(\bar{x})$. The stated equality follows. From [5, Exercise 8.14 \& Proposition 8.12], $\partial \delta_{C \cap L_{\bar{x}}}(\bar{x})=$ $\partial^{\infty} \delta_{C \cap L_{\bar{x}}}(\bar{x})=\left[\widehat{\partial} \delta_{C \cap L_{\bar{x}}}(\bar{x})\right]^{\infty}$. Thus,

$$
\partial \delta_{C \cap L_{\bar{x}}}(\bar{x})=\partial^{\infty} \delta_{C \cap L_{\bar{x}}}(\bar{x})=\left[\widehat{\partial} \delta_{C \cap L_{\bar{x}}}(\bar{x})\right]^{\infty}=\partial^{\infty}\left(\delta_{C}+h\right)(\bar{x}) .
$$

Together with (7), we obtain the conclusion for $h(\cdot)=v\|\cdot\|_{0}$. By following the same arguments as above, one may obtain the second part.

\section{Conclusion}

Since [6, Proposition 3.2] is only used to check [6, Assumption 4.1(iii)], the results in [6, Section 4] are all correct by Proposition 2.1.

Acknowledgements Funding was provided by National Natural Science Foundation of China (Grant Nos. 11971177 and 11701186).

\section{References}

1. Ioffe, A.D., Outrata, J.V.: On metric and calmness qualification conditions in subdifferential calculus. Set Valued Var. Anal. 16, 199-227 (2008)

2. Mordukhovich, B.S.: Variational Analysis and Generalized Differentiation I: Basic Theory. Springer, Heidelberg (2006)

3. Mordukhovich, B.S.: Variational Analysis and Applications. Springer, Cham (2018)

4. Robinson, S.M.: Some continuity properties of polyhedral multifunctions. Math. Program. Stud. 14, 206-214 (1981)

5. Rockafellar, R.T., Wets, R.J.: Variational Analysis. Springer, New York (1998)

6. Wu, Y.Q., Pan, S.H., Bi, S.J.: Kurdyka-Lojasiewicz property of zero-norm composite functions. J. Optim. Theory App. 188, 94-112 (2021)

Publisher's Note Springer Nature remains neutral with regard to jurisdictional claims in published maps and institutional affiliations. 